\title{
CONTEMPORARY RESULTS OF HUMAN MICROBIOME STUDIES WITH PARTICULAR EMPHASIS PLACED ON THE ORAL MICROBIOME AND ITS ROLE IN ETIOPATHOGENESIS OF PERIODONTITIS
}

\author{
Maria Wiernicka-Menkiszak', Elżbieta Dembowska', Beata Kozłowska² \\ 'Department of Periodontology, Pomeranian Medical University, Szczecin, Poland \\ ${ }^{2}$ Department of Radiology and Radiovisiography, Provintional Hospital, Szczecin, Poland
}

\begin{abstract}
In 2005 a broad scale project on human microbiome research (the Human Microbiome Project - HMB) was initiated. Its aim was to research the composition of the microflora of the human body, and create a genome database of microorganisms existing on the human body surface and having contact with the external environment. The scope of the project involved examining oral microbiomes of 250 healthy individuals at reproductive age. As a result, an extensive database of bacterial genomes was created and variabilities dependent on the examined environment, race, sex, ethnicity, and eating habits were determined.

The aim of this paper is to present the contemporary state of knowledge on the composition of microorganisms populating the human body with a special emphasis placed on the composition of oral microflora and subgingival biofilm, and including comments regarding phylogenetic adaptation of humans to life in the world of microorganisms.

Contemporary methods of microorganism identification significantly broaden our knowledge on the composition of microflora in various biotopes, and correlations of microorganisms in different ecosystems as well as among each other. However, the knowledge is still fragmented and fails to provide a firm basis to introduce advantageous and permanent changes in the microbiome. Yet, it allows for verifying some hitherto accepted theories regarding the role of bacteria in health and sickness. Thanks to the above, in periodontology views on etiopathogenesis of periodontitis have been changing.

The contemporary state of knowledge on the human oral microbiome is becoming broader and broader. It may lead to discussions about changing some hypotheses regarding the role bacteria play in periodontitis; however, there are too many unknowns to provide bases for pharmacological or biological changes in its composition.
\end{abstract}

KEY WORDS: human microbiome, oral microbiome, phylogenetic adaptation, periodontitis.

J Stoma 2018; 71, 6: 502-506

DOI: https://doi.org/10.5114/jos.2018.85567

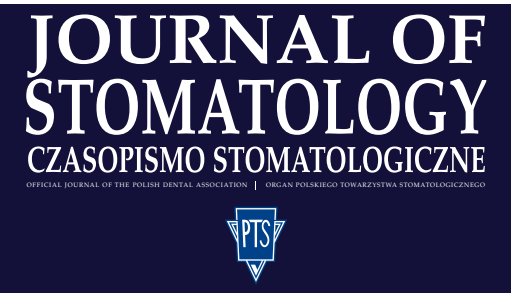

AdDress For CORRESPONDENCE: dr n. med. Maria Wiernicka-Menkiszak, Department of Periodontology, Pomeranian Medical University,

Al. Powstańców Wlkp. 72, 70-111 Szczecin, Poland, phone: +48 509564 589, e-mail: maria.wiernicka@poczta.onet.pl 


\section{INTRODUCTION}

In 1990 the United States Department of Energy (DOE) and the United States National Institutes of Health (NIH) awarded the funds for conducting the Human Genome Project (HGP), and since that time there has been incredible progress in techniques of automatic DNA sequencing. The result of the research was not only the creation of an initial human genome map published in 2003, but also lowering the cost of used DNA sequencing methods [1]. It allowed for broad scale detection of DNA in the human body that did not originate in the Homo genus, and then, in 2005 led to initiation of a project aiming at researching genomes of microorganisms populating the human body, i.e. the Human Microbiome Project (HMP) [2-4].

The first such broad research project focused on the human microbiome was performed between 2005 and 2011. Its objective was to examine microbiomes from 250 individuals in good general health. The main aim was to enrich the HMP database with several hundred bacterial genomes, and determine relationships between changes in microbiomes and being healthy or ill. According to the grant's provision, the research was time-bound and thus it was not possible to confirm a causal connection between microorganisms and the condition of the host's body [4]. A long-term objective of the human microbiome studies is its control, creating the basis for the control and management of human bacterial flora [5].

The aim of this paper is to present the results of the latest studies regarding the composition of microflora populating the human body with particular emphasis placed on the oral microbiome, and supplement this knowledge with the basis of phylogenetic adaptation of humans to life in the world of microorganisms.

\section{MICROBIOME}

Joshua Lederberg offered the term "microbiome" at the turn of the $21^{\text {st }}$ century [6]. As distinct from microbiota, the microbiome is considered to include all organisms populating a given biotope (habitat), with the exception of pathogenic bacteria [6]. Currently, the term is used not with regard to microorganisms, but rather to their genome [7].

Between 2006 and 2011 the Human Microbiome Project involved examination of 300 microbiomes of healthy adult White and Latino individuals (151 women and 149 men) aged 18-40 years old who lived in two American states: Texas and Missouri [2]. Samples from the oral cavity, nostrils, epidermis, distal intestine, and vagina were collected twice in most cases every 35-404 days, and sometimes even three times. DNA of microorganisms was cloned and sequenced by means of bacterial genes' $16 \mathrm{~S}$ rRNA. Studies of the human microbiome showed that even healthy individuals differ significantly in terms of microorganisms populating the above-mentioned kinds of habitats. Although many bacterial species were revealed in all examined individuals, qualitative composition of microflora depended on race, ethnicity, place of residence, eating habits, or early exposure to microorganisms.

The strongest connections in bacterial genomes were observed with regard to ethnicity and race. The biggest differences were noted between microbiomes of males and females. Within one sex microbiome variants were not so different. Intestinal and oral flora was the most ecologically diverse. The least varied was the vaginal microbiome. Changes in skin microbiome were greatly dependent on age of the examined individuals. Interestingly, out of 327 bacteria hitherto considered being "pathogens", 56 were found in the microbiome of healthy individuals - all opportunistic and widespread within the evaluated habitats. Thus, a hypothesis was formed that expression in the human microbiome may be analogous to expression of genetic features hidden in recessive alleles - it is triggered by environmental changes. Given that, metabolism of the host is an important factor for the human microbiome $[3,4]$.

Scientific progress with regard to identifying genomes is so great that Aagaard et al's studies [4] from 2011 ceased to be the only source of knowledge on the human microbiome. They could not be exhaustive since only some and the most accessible regions of the human body were planned to be examined. Dynamics of modern research allows for discovering and publicizing more and more new bacterial, viral, or fungal genomes populating the human body.

Kovarsky et al. broadened the scope of human microbiome research with genomes of bacteria, viruses, and other DNA particles circulating in peripheral blood of individuals who have undergone organ transplants [8]. Consequently, they enriched the database including approximately 8,000 genomes with over 1,000 new units. Bacterial genomes constituted two-thirds of them; the rest belonged to viruses and fungi [8].

Development of DNA sequencing methods allowed it to be proved that also a healthy human fetus has contact with bacteria, even before it leaves the intrauterine environment. The meconium of healthy neonates born at term was confirmed to have bacterial DNA that exhibited significant similarities to bacterial DNA found in the oral cavity of the mother. [9]

The observations shed a completely different light on the role of microorganisms in the human body. Bacteria as parasitic organisms requiring external identification and absolute control (antibiotics, chemotherapeutic agents) start to be seen as organisms indispensable for normal functioning of the human body. By exhibiting mutualism and competitiveness, they become a functionally integral part of the human body. The latest studies of oral microflora led to changes in views regarding etiopathogenesis of periodontitis. 


\section{ORAL MICROBIOME AND DENTAL BIOFILM BACTERIA}

Till 2001, Human Oral Microbiome Database (HOMD) included genetic sequences of 700 different taxonomic units belonging to 14 biological types $[10,11]$. The Human Microbiome Consortium examined the oral microbiome in 242 healthy individuals (129 men and 113 women) with regard to nine oral habitats. They included: saliva, buccal mucosa, keratinized gingiva, palate, tonsils in the throat, dorsal surface of the tongue, and supra- as well as subgingival biofilm. Considering the examined habitats, bacterial flora was the most ecologically diverse in the saliva. Despite the great diversity of microorganisms, their specific composition in the examined individuals within one population was similar. Most oral habitats were dominated by Streptococcus species with some exceptions: Haemophilus dominated in the buccal mucosa, Actinomyces species were predominant in supragingival plaque, whereas Prevotella species were predominant in the subgingival plaque. Differences between particular studies proved to be specific, significant, and personalized [3].

A multitude of planktonic bacteria does not cause health problems in edentulous individuals. It is a biofilm forming on the teeth surface (enamel, cementum) that is a problem since it facilitates creation of a new ecosystem for microorganisms: an ecosystem in which trophic dependencies and co-operation between the microorganisms limit the host's abilities for successful defense.

Results of past studies showed the presence of 41 bacterial species in the subgingival plaque. Socranscy et al. observed that the bacterial spectrum of dental biofilm in individuals with both healthy periodontium and all forms of periodontitis is similar, but the number of particular types of bacteria varies [12]. The following three Gram-negative anaerobia dominated in the deep periodontal pockets: Porphyromonas gingivalis, Tannerella forsythia, and Treponema denticola. Consequently, a theory of "red complex" and the term "periopathogen" emerged that did not meet Koch's postulates [12-14]. Recent studies indicate that the spectrum of types and species of microorganisms present in subgingival biofilm is much wider.

So far, 102 genera and 203 species of bacteria populating periodontal pockets in chronic periodontitis have been identified. To those earlier detected microorganisms the following bacterial genera should be added: Anaeroglobus (especially A. geminatus), Capnocytophaga gingivalis, TM7, and Veilonella sp. More advanced inflammations revealed Desulfobulbusa propionicus, Eubacterium saphenum, Filifactor alocis, Streptococcus constellatus, Tannerella forsythia, Treponema denticola, Peptostreptococcus stomatis, Megasphera sp., Selenomonas sp., Dialister sp., and Synergistesten sp. [15, 16]. Their role in periodontitis is important. Furthermore, it was shown that the common belief that Gram-negative bacteria prevail in the subgingival biofilm during the course of periodontitis is false. In very deep periodontal pockets, Gram-positive anaerobia also exhibit significant growth in their numbers $[17,18]$. Heterogeneity of the subgingival microbiome in the pocket higher than previously thought; presence of bacteria considered to be "periopathogens" in healthy periodontium, no possibility to remove them permanently from the oral habitat, and co-variability regarding incidence of newly identified bacteria with a periodontitis phenotype change the existing understanding of the etiology of this disease [16, 19-23].

There is an ongoing discussion whether the "red complex" model should be maintained and the role of three "periopathogens" acknowledged as superior, or a new model for periodontitis based on dysbiosis should be accepted. An etiopathogenesis model proposed by Hajishengallis and Lamont assumes that periodontitis is not caused by activity of chosen "periopathogens", such as "red complex", but is a consequence of synergic activity of biofilm microorganisms leading to dysbiosis in the habitat of a periodontal pocket (polymicrobial synergy and dysbiosis model; PSD model) [24]. It was observed that some bacteria, e.g. Filifactor alocis, which usually exhibit mutualism, in conditions of oxidative stress change the qualities of the subgingival plaque habitat in such a way that they allow for Porphyromonas gingivalis species' penetration into the epithelium of a pocket [17].

The fact that certain biofilm bacteria may cross the epithelial border and permeate into the gingival tissues has been known since 1965 [25]. An analysis of 440 articles obtained from Medline-PubMed, CochraneCENTRAL, ISI Web of Knowledge, and SCOPUS on the possibility of subgingival biofilm bacteria invasion of the epithelial pockets was conducted. Due to methodological reasons, only three studies qualified for the final analysis. Only one study showed statistically significant differences in the presence of Aggregatibacter actinomycetemcomitans, Tannerella forsythia, Prevotella intermedia, Campylobacter ochracea, and Campylobacter rectus in periodontal pockets and healthy gingival crevice. In the other ones, such a significant difference was not observed. It demonstrates that currently available studies on the ability of subgingival biofilm microorganisms to penetrate periodontal tissues are of poor or medium quality, are inconsistent, differ in methodologies used, and the research projects are poorly documented. Hence, they fail to provide sufficient evidence to support or reject a hypothesis regarding the invasion of pathogens into periodontal tissues as a key pathomechanism in etiopathogenesis of periodontitis [26].

\section{PHYLOGENETIC ADAPTATION OF HUMANS TO LIVE IN THE WORLD OF MICROORGANISMS}

Our planet is considered to be 4.5 billion years old [27]. Available knowledge on the existence of the first 
organism, a prokaryote, able to self-duplicate, indicated the time prior to 3.5 billion years [28]. Seven million years ago in Africa there lived a hominid that is considered to be the last animal ancestor of the human species by anthropologists - Sahelanthropus tchadensis. A species that could be classified as Homo appeared 2 million years ago, while Homo erectus appeared 1.5 million years ago $[29,30]$. It means that when humans started to colonize the Earth, bacteria had already populated all the places for 3.5 billion years. The process of life development on our planet was not granted, and there was a lot of time to select specimens that were adapted to the conditions of the external environment. It was the Homo species that entered the world of bacteria, and not the other way round. This fact also explains why we became a biotope (habitat) for bacteria.

A proof of our adaptation to life in the world of bacteria is an observation regarding intrauterine life in the fifth week of embryonic life (counting from conception), when myelopoiesis starts in the yolk sac attached to a human embryo. At that time, the first monocytes (macrophages) and microphages appear - the cells that constitute the basis for natural immunity and use the most effective mechanism to eliminate bacteria known in nature, i.e. phagocytosis. Myelopoiesis starts before the embryo is connected to the mother's body via the umbilical cord [31]. After birth, the human body is additionally protected by endothelia, whose continuity prevents the organism from being invaded by bacteria, $\mathrm{pH}$ of skin and mucosal secretions as well as by antibacterial (lysozyme, phospholipase, defensins, transferrin, and lactoferrin) antifungal (chitinase), and antiviral (interferon) substances they contain.

Adaptive immunity, present only in vertebrates, is conditioned by a small retrosternal, central lymphoid organ, i.e. the thymus. The thymus reaches its full maturity during the second year of life of a child. When an individual reaches sexual maturity, the organ gradually involutes. At that time, it is responsible for the entire process of acquiring competence by thymus-dependent lymphocytes (T-lymphocytes), i.e. shaping the major histocompatibility complex (MHC; human leucocyte antigens - HLA), and forming adaptive immunity [32]. Thanks to these mechanisms, despite the fact that there are 700 different bacterial taxonomy units within the oral cavity, dentists do not observe infections after performing procedures that break the continuity of tissues in individuals with functioning immune systems, even during the course of the following autoimmune diseases: pemphigus, pemphigoid, and erosive or follicular lichen planus (Figure 1). The phylogenetic adaptation of a human body to live in symbiosis with bacteria is so great that during bacteremia, which always occurs during meals, even at the first bite the vegetative system acts on the immune system, and leads to activation of wall neutrophils, which may increase the number of neutrophilic granulocytes circulating in the blood by $30 \%$ [32].

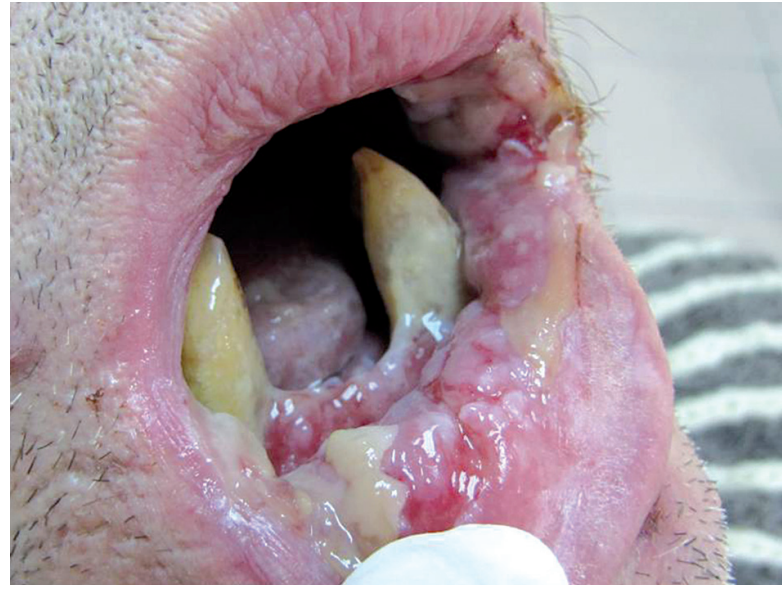

FIGURE 1. A 68-year-old patient diagnosed with pemphigus vulgaris. Lesion in the oral mucosa are characterised by extensive erosions with visible epithelium of damaged blisters. Hygiene of residual dentition is not preserved. No sings of bacteraemia. Clinical examination did not confirm an increased body temperature; group B submandibular lymph nodes were palpable, not tender, single and movable; submental lymph nodes were non palpable and not tender. CBC findings were normal

\section{CONCLUSIONS}

Thanks to the formed adaptive and competitiveness mechanisms of microorganisms in the environment, healthy individuals are rarely populated by pathogenic bacteria. Intestinal bacteria are necessary to break down food, they co-participate in vitamin production, and the relations between a healthy human body and its microflora are of symbiotic nature (mutualism). Perceiving bacteria as pathogens only is wrong, and highly civilized populations have paid for this view with widespread dysbioses (Clostridium difficile colitis; hospital-acquired infections), and allergies involving skin, digestive system as well as the respiratory system. Abusing antibiotics in combating bacteria led to the emergence of strains that are resistant to know antibacterial drugs. The World Health Organization published a list of twelve bacteria for which new antibiotics are urgently needed: priority 1 (critical) includes Acinetobacter baumannii, Pseudomonas aeruginosa, and Enterobacteriaceae sp.; priority 2 (high) includes Enterococcus faecium, Staphylococcus aureus, Helicobacter pylori, Campylobacter jejuni, Salmonellae sp., and Neisseria gonorrhoeae; priority 3 (medium) includes Streptococcus pneumoniae, Haemophilus influenzae, and Shigella spp. All listed microorganisms constitute opportunistic microflora that is widespread in nature [33].

The human genome is $98-99 \%$ compatible. The remaining $1-2 \%$ constitutes the variabilities resulting from mutations that aim at phylogenetic adaptation to life in a given environment [4]. The genotype of microflora is even more difficult to examine due to fast adaptive 
processes of microorganisms and formation of a vast number of mutations. Perhaps, due to dynamics of life processes in the world of microorganisms, determining the present-day microbiome of the Earth and its inhabitants may never be possible. However, determining metabolic dependencies of microorganisms in biotopes and co-dependencies between various microbiomes constitutes an even greater challenge. Therefore, special caution is advised in introducing new technologies and treatment methods based on detailed molecular diagnostics that fail to treat a human organism as a whole.

\section{CONFLICT OF INTEREST}

The authors declare no potential conflicts of interest with respect to the research, authorship, and/or publication of this article.

\section{References}

1. Abecasis GR, Auton A, Brooks LD, et al. An integrated map of genetic variation from 1.092 human genomes. Nature 2012; 491: 56-65.

2. Peterson J, Garges S, Giovanni M. The NIH Human Microbiome Project. Genome Res 2009; 19: 2317-2323.

3. The Human Microbe Consortium. Structure, function and diversity of the healthy human microbiome. Nature 2012; 486: 207-214.

4. Aagaard K, Petrosino J, Keitel W, et al. The Human Microbe Projects for compressive sampling of the human microbiome and why it matters. FASEB J 2013; 27: 1012-1022.

5. NIH HMP Working Group, Peterson J, Garges S, Giovanni M, et al. The NIH Human Microbiome Poject. Genome Res 2009; 19: 2317-2323.

6. Lederberg J, McCray AT. 'Ome sweet 'omicis - a genealogical treasury of words. Scientis 2001; 15: 8 .

7. Gill SR, Pop M, Beboy RT, et al. Metagenomic analysis of the human distal gut microbiome. Science 2006; 312: 1355-1359.

8. Kowarsky M, Camunas-Soler J, Kertesz M, et al. Numerous uncharacterized and highly divergent microbes witch colonize humans are revelated by circulating cell-free DNA. Proc Natl Acad Sci U S A 2017; 114: 9623-9628.

9. Prince AL, Antony KM, Aagaard K. The microbe and development: a mothers perspective. Semin Report Med 2014; 32: 14-22.

10. Human Oral Microbiology. Encyclopedia of Metagenomics 2015; 271-291.

11. Paster BJ, Boches SK, Galvin JL, et al. Bacterial diversity in human subgingival plaque. J Bacteriol 2001; 183: 3770-3783.

12. Socranscy S, Haffajee AD, Cugini MA, et al. Microbial complexes in subgingival plaque. J Clin Periodontol 1998; 25: 134-144.

13. Socransky SS, Haffajee AD. Dental biofilms: difficult therapeutic targets. Periodontol 2000 2002; 28: 12-55.

14. Socranscy SS, Haffajee AD. Periodontal microbial ecology. Periodontology 2000, 2005; 38: 135-187.

15. Camelo-Castillo A, Novoa L, Balsa-Castro C, et al. Relationship between periodontitis-associated subgingival microbiota and clinical inflammation by $16 \mathrm{~S}$ pyrosequencing. J Clin Periodontol 2015; 42: 1074-1082.

16. Griffen AL, Beall CJ, Campbell JH, et al. Distinct and complex bacterial profiles in human periodontitis and heath reelevated by pyrosequencig. ISME J 2012; 6: 1176-1185.

17. Moffat CE, Whitemore SE, Griffen AL, et al. Filifactor alocis interactions with gingival epithelial cells. Mol Oral Microbiol 2011 26: 365-373.

18. Aruni AW, Roy F, Fletcher HM. Filifactor alocis has virulence attributes that can enhance its persistence under oxidative stress conditions and mediate invasion of epithelial cells by Porphyromonas gingivalis. Infect Bacteriol 2011; 79: 3872-3886.

19. Ximenez-Fyvie LA, Haffajee AD, Socransky SS. Microbial compositions of supra- and subgingival plaque In subjects with adult periodontitis. J Clin Periodontol 2000; 27: 722-732.

20. Mayanagii G, Sato T, Shimauchi H, Takahashi N. Detection frequency of periodontitis-associated bacteria by polymerase chain reaction in subgingival and supragingival plaque of periodontitis and healthy subjects. Oral Microbiol Immunol 2004; 1: 379-385.

21. Diaz PI, Chalmers NI, Rickard AH, et al. Molecular characterization of subjects-specific oral microflora during initial colonization of enamel. Appl Environ Microbiol 2006; 72: 2837-2848.

22. Dewhirst FE, Chen T, Izard J, et al. The human oral microbiome. J Bacteriol 2010; 192: 5002-5017.

23. Curtis MA, Zenobia C, Darveau DR. The relationship of the oral microbiota to periodontal health and disease. Cell Host Microbe 2011; 10: 302-306.

24. Hajishengallis G, Lamont RJ. Beyond the red complex and in more complexity: the polymicrobial synergy and dysbiosis (PSD) model of periodontal disease etiology. Mol Oral Microbiol 2012; 27: 409-419.

25. Listgarten MA. Electron microscopic observations on the bacterial flora of acute necrotizing ulcerative gingivitis. J Periodontol 1965; 36: 328-339.

26. Mendels L, Azevedo NF, Felino A, et al. Relationship between invasion the periodontium by periodontal pathogens and periodontal disease: a systematic review. Virulence 2015; 6: 208-215.

27. New image of infant universe reveals era of first stars, age of cosmos and more. NASA, 2003.02.11.

28. Penny D, Poole A. The nature of the last Universal common ancestor. Curr Opin Gen Dev 1999; 9: 672-677.

29. Goren-Inbar N, Alperson N, Kislev ME, et al. Evidence of hominin control of fire at Gesher Benot Yaaqov, Israel. Science 2004; 304: 725-727.

30. Gibbons A. Paleoanthropology. Oldest members of Homo sapiens discovered in Africa. Science 2003; 300: 1641.

31. Steinborn A, Sohn C, Sayehli C, et al. Spontaneous labor at term is associated with fetal monocyte activation. Clin Exp Immunol 1999; 11: 147-152.

32. Zabel M. Histologia. Urban \& Partner, Wrocław 2004; 85, 130-135.

33. World Health Organization. Global priority list of antibioticresistant bacteria to guide research, discovery, and development of new antibiotics, 2017. 